\title{
ITERATIVE PROJECTION AND REFLECTION METHODS: THEORY AND PRACTICE
}

\author{
MATTHEW K. TAM
}

(Received 15 March 2016; first published online 18 May 2016)

2010 Mathematics subject classification: primary 90C30; secondary 49M37.

Keywords and phrases: feasibility problem, projection algorithm, Douglas-Rachford algorithm, projection operator, reflection operator.

The focus of this thesis is the family of so-called projection and reflection methods. These methods form the basis for a class of iterative algorithms which can be used to solve the feasibility problem, that is, the problem of finding a point in the intersection of a collection of constraint sets. Many optimisation and reconstruction problems can be profitably modelled within this framework, although the formulation is not always immediately obvious. In a typical feasibility problem the target intersection set is difficult to deal with directly, and projection and reflection algorithms overcome this by exploiting relatively simpler structure in each of the individual constraint sets from the collection. From the perspective of the practitioner, the methods are appealing due to their relative simplicity, ease-of-implementation and empirical good performance.

In recent times, a particular member of the family, the Douglas-Rachford method, has received special attention. This is, in part, due to its striking ability to successfully solve a variety of difficult nonconvex problems including those of a combinatorial nature. From a theoretical perspective, the lack of a sound theoretical foundation to justify and to explain such empirical successes begs for further investigation.

The organisation of the thesis is as follows: Chapter 1 introduces basic definitions, notation and background, before formally introducing the feasibility problem framework and fundamental projection-type algorithms. Chapter 2 focuses on theory in the presence of convex constraint sets, and introduces the recently developed cyclic Douglas-Rachford method. Chapter 3 focuses on theory in the absence of convexity. In this case, theoretical underpinnings are still in development and rather more cumbersome. Specific classes or instances of nonconvex feasibility problems must be considered separately. Chapter 4 investigates applications, particularly of the

Thesis submitted to the University of Newcastle in December 2015; degree approved on 3 March 2016; supervisors Jonathan M. Borwein, Regina S. Burachik and Brailey Sims.

(C) 2016 Australian Mathematical Publishing Association Inc. 0004-9727/2016 \$16.00 
Douglas-Rachford algorithm to settings without convexity. Chapter 5 indicates open problems for future research.

The main contributions of the thesis can be summarised as follows.

- The discovery and analysis of the cyclic Douglas-Rachford method [2, 3], in the convex setting; the first many-set extension of the classical Douglas-Rachford method not requiring a product-space reformulation.

- A thorough study of the local regularity properties of sparse nonnegative vector and low-rank positive-semi-definite matrix constraints. A knowledge of these properties is of fundamental importance, for instance, in justifying the application of the Douglas-Rachford method to problems involving low-dimensional distance matrix reconstruction.

- The first global convergence results for the classical Douglas-Rachford method which apply to problems of a combinatorial nature. These results complement the emerging local theory [4] as well as being of interest in their own right.

- An extensive empirical investigation of nonconvex problems which the DouglasRachford method can successfully solve [1]. This growing library of problems is important both for the refinement of applications and to guide theoretical developments.

\section{References}

[1] F. J. Aragón Artacho, J. M. Borwein and M. K. Tam, 'Douglas-Rachford feasibility methods for matrix completion problems', ANZIAM J. 55(4) (2014), 299-326.

[2] J. M. Borwein and M. K. Tam, 'A cyclic Douglas-Rachford iteration scheme', J. Optim. Theory Appl. 160(1) (2014), 1-29.

[3] J. M. Borwein and M. K. Tam, 'The cyclic Douglas-Rachford method for inconsistent feasibility problems', J. Nonlinear Convex Anal. 16(4) (2015), 537-584.

[4] H. M. Phan, 'Linear convergence of the Douglas-Rachford method for two closed sets', Optimization 65(2) (2016), 369-385.

MATTHEW K. TAM, Institut für Numerische und Angewandte Mathematik, Universität Göttingen, 37083 Göttingen, Germany

e-mail:m.tam@math.uni-goettingen.de 